\title{
MODULATOR SYSTEMS FOR THE ESS 2.5 MeV FAST CHOPPER
}

\author{
Michael A. Clarke-Gayther, Rutherford Appleton Laboratory, Didcot, United Kingdom
}

\section{Abstract}

Modulator system requirements for the ESS $2.5 \mathrm{MeV}$ slow wave chopper are considered to be challenging. A description is given of candidate modulation schemes, and modulator configurations, where the chopper E-field is generated by a gated high frequency sinusoidal / trapezoidal waveform or by a combination of fast transition short duration, and slow transition long duration, trapezoidal pulses.

\section{INTRODUCTION}

The European Spallation Source (ESS) [1], is the most ambitious of the existing proposals for the next generation of accelerator driven pulsed neutron sources [2]. Designed to address the rapid expansion in the field of condensed matter research, the ESS accelerator will generate intense, fast pulsed, beams of neutrons, by delivering up to $5 \mathrm{MW}$ of protons to a liquid mercury (4 MW), and solid tantalum (1 MW) target, at a pulse repetition frequency (prf) of 40 and $10 \mathrm{~Hz}$, respectively.

\section{ESS 2.5 MeV FAST CHOPPER}

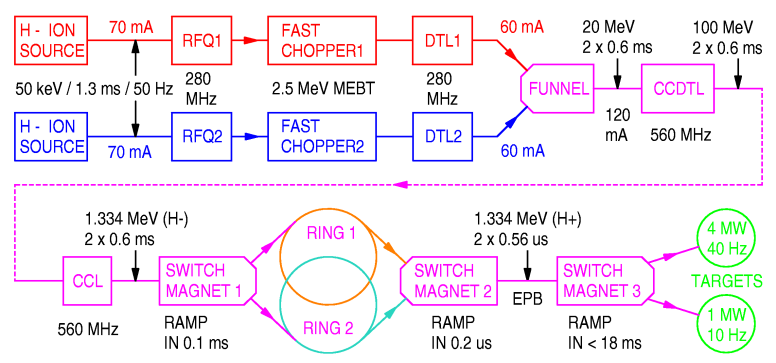

Figure 1: ESS block schematic

Components of the ESS, are shown in schematic form in figure 1. The low beam loss $(<1 \mathrm{nA} / \mathrm{m} @ 1.334 \mathrm{MeV})$ design features a high current $\mathrm{H}$ - linac, and twin accumulator rings with sequential charge exchange injection and sequential fast extraction.

The time dependant function of the fast choppers, switching, and fast extraction magnets, is shown in figure 2, where the 'history' of a beam pulse is traced from RFQ to target, over a linac cycle. The seven chopp-ing regimes listed in figure 2 , are described in Table 1.

Stringent beam loss requirements dictate that the chopping field in the $2.5 \mathrm{MeV}$ medium energy beam transport line (MEBT), rise and fall (1 to $90 \%, 90$ to $1 \%$, for $<2 \%$ transverse emittance growth) within the beam bunch interval of $2.9 \mathrm{~ns}$. In addition, the chopping field duration must be instantaneously programmable in the range $240 \mathrm{~ns}-0.1 \mathrm{~ms}$ (See Table 1). Slow wave (E-field) transmission line structures have demonstrated field

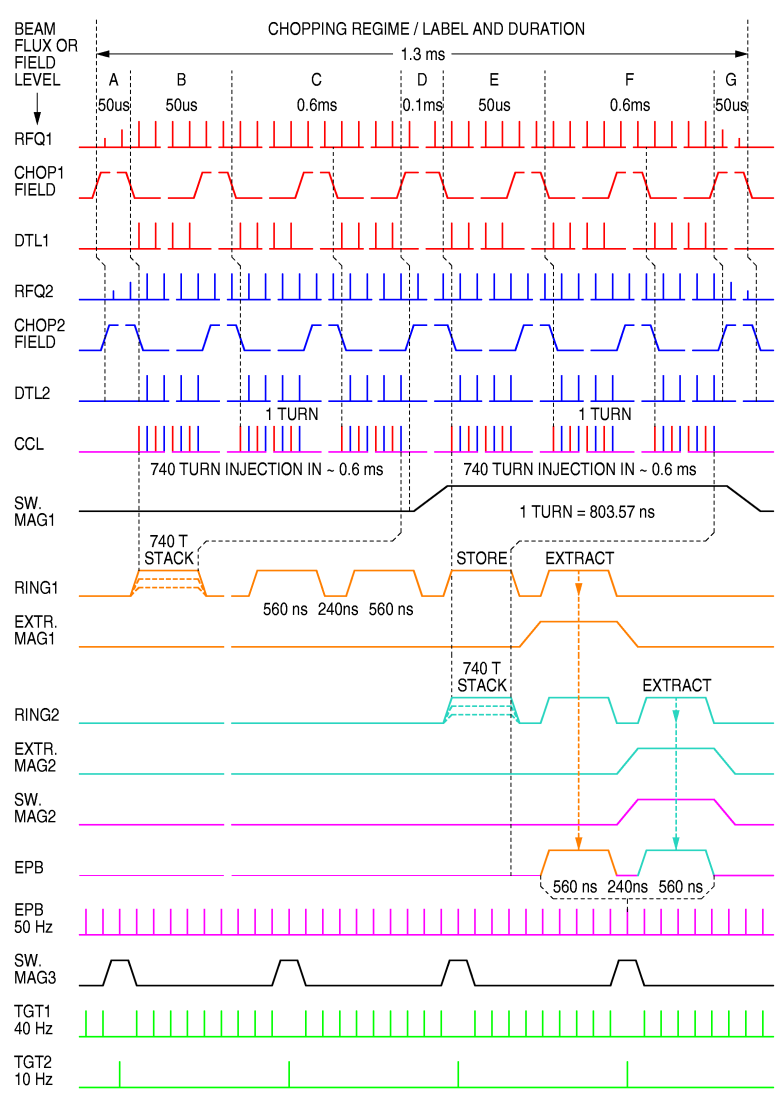

Figure 2: ESS beam chopping and switching

transition times in the nanosecond regime [3, 4,], but the requirement for a fast transition time, long pulse duration, high voltage $(\sim 2.5 \mathrm{kV})$, high burst frequency modulator, is challenging.

Table 1: ESS chopping regimes

\begin{tabular}{|c|c|c|c|c|c|c|}
\hline \multirow{2}{*}{\begin{tabular}{|r} 
Label \\
fig.2 \\
\end{tabular}} & \multirow{2}{*}{ Chopping Regime } & \multirow{2}{*}{$\begin{array}{l}\text { Dura- } \\
\text {-tion }\end{array}$} & \multicolumn{2}{|c|}{ Chopper Duty Cycle } & \multirow{2}{*}{$\begin{array}{l}\text { No. of } \\
\mid \text { Turns } \\
\end{array}$} & \multirow[t]{2}{*}{ Function } \\
\hline & & & On time & Off time & & \\
\hline$A$ & Ion source transition & $50 \mu \mathrm{s}$ & $50 \mu s$ & 0 & 60 & Gates low intensity beam \\
\hline$B$ & $\begin{array}{l}\text { Beam duty cycle } \\
\text { ramping }\end{array}$ & $50 \mu \mathrm{s}$ & $\begin{array}{l}803.57 \mathrm{~ns} \\
\text { to } 241.1 \mathrm{~ns} \\
\end{array}$ & $\begin{array}{r}0 \text { to } \\
562.5 \mathrm{~ns} \\
\end{array}$ & 60 & $\begin{array}{c}\text { Limits linac beam loading } \\
\text { transient }\end{array}$ \\
\hline C & Ring 1 stacking & $0.6 \mathrm{~ms}$ & $241.1 \mathrm{~ns}$ & $562.5 \mathrm{~ns}$ & 740 & Gaps for fast extraction \\
\hline $\mathrm{D}$ & Ring switching & $0.1 \mathrm{~ms}$ & $0.1 \mathrm{~ms}$ & 0 & 120 & Gap for ring switching \\
\hline$E$ & $\begin{array}{l}\text { Beam duty cycle } \\
\text { ramping }\end{array}$ & $50 \mu \mathrm{s}$ & $\begin{array}{l}803.57 \mathrm{~ns} \\
\text { to } 241.1 \mathrm{~ns} \\
\end{array}$ & $\begin{array}{r}0 \text { to } \\
562.5 \mathrm{~ns} \\
\end{array}$ & 60 & $\begin{array}{c}\text { Limits linac beam loading } \\
\text { transient }\end{array}$ \\
\hline $\mathrm{F}$ & Ring 2 stacking & $0.6 \mathrm{~ms}$ & $241.1 \mathrm{~ns}$ & $562.5 \mathrm{~ns}$ & 740 & Gaps for fast extraction \\
\hline G & Ion source transition & $50 \mu \mathrm{s}$ & $50 \mu \mathrm{s}$ & 0 & 60 & Gates low intensity beam \\
\hline
\end{tabular}

\section{CANDIDATE MODULATION SCHEMES}

Two candidate modulation schemes are presented. Both schemes demonstrate a new 'periodic' transverse beam kicking technique that should find application in chopping and funnelling systems. The conflicting transit-ion time and pulse duration requirements are resolved by making use of the periodic nature of the beam. 


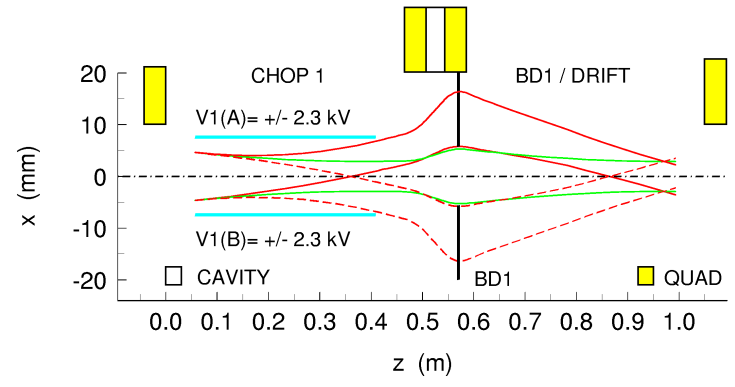

Figure 3: Scheme A / Beam trajectories

Unipolar, long duration fields are replaced by bipolar (sinusoidal or trapezoidal pulsed) slow wave fields at half linac frequency, and the gated RF amplifier, bipolar pulse generator, and transmission line transformer, become the components of choice for modulator design.

\subsection{Modulation scheme A}

Dimensions, and beam trajectories for modulation scheme A are shown in figure 3, where the chopped and un-chopped beams are shown in red and green respectively. The beam trajectories are beam envelopes containing $100 \%$ of beam particles, and include the effects of space charge and image forces [6].

A timing and layout schematic for scheme $\mathrm{A}$ is shown in figure 4 , where two slow wave electrodes (A, and B) produce a bipolar pulsed field at half linac frequency, that deflects alternate bunches through $\pm 16.5 \mathrm{mr}$, to beam dump (BD1), for the total duration of the chopped beam interval. Each modulator must deliver a peak power of $\sim 53 \mathrm{~kW}(1.65 \mathrm{~kW}$ mean) into a $50 \mathrm{Ohm}$ load. BD1 must dissipate a total beam power of $\sim 4.7 \mathrm{~kW}$.

\subsection{Modulation scheme B}

Beam trajectories, and a timing schematic for scheme B are shown in figures 5 , and 6 respectively, where slow wave chopper 1 , produces a bipolar, pulsed field that deflects just two adjacent bunches through $\pm 16.5 \mathrm{mr}$, to beam dumps 1 and 2 (BD1, BD2), creating two $10 \mathrm{~ns}$ duration gaps in the bunch train, at the beginning and end of each chopped beam interval. Modulator peak power at $\sim 35 \mathrm{~kW}$ ( $\sim 33 \mathrm{~W}$ mean!), is much lower than scheme A.

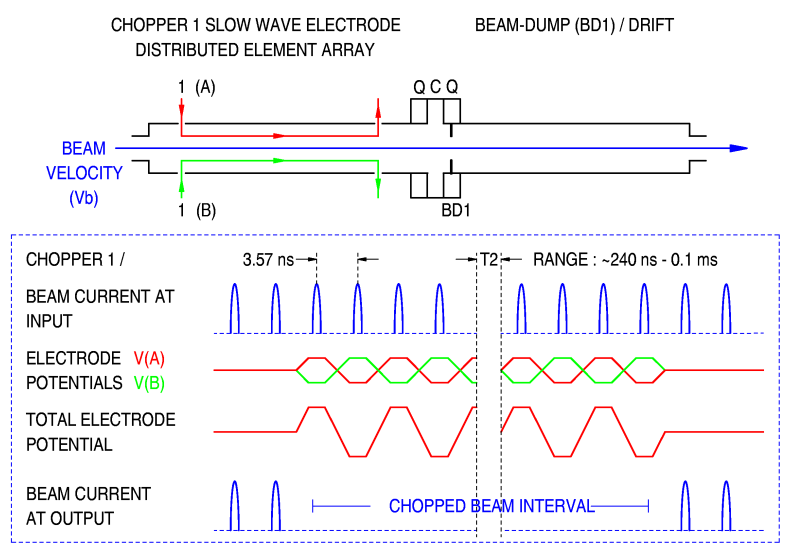

Figure 4: Scheme A / Timing schematic

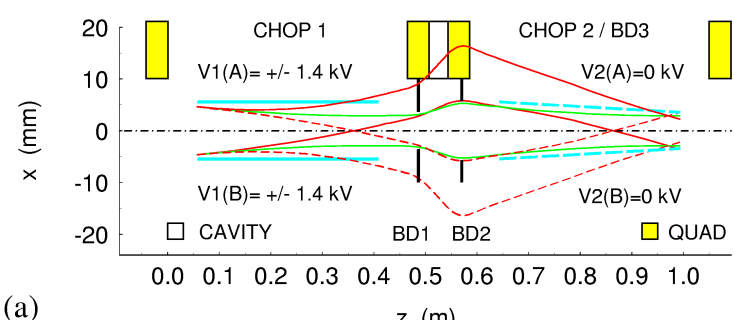

(a)

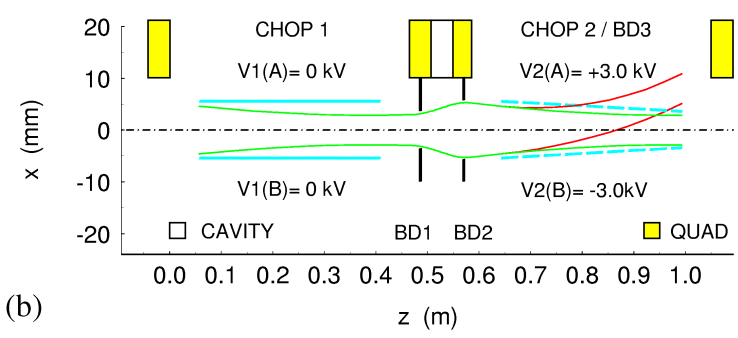

Figure 5: Scheme B / Beam trajectories

(a) Fast transition pre-chopping - beam to BD1\&2.

(b) Slower transition chopping - beam to BD3.

The chopper 2, slow wave, lumped element array ( 6 pairs of $6 \mathrm{~cm}$ long electrodes), produces a unipolar, pulsed field that deflects the beam through $47 \mathrm{mr}$ to a water cooled electrode / beam dump array (BD3).

The chopper 2 modulators ( $12 \mathrm{x}$ switch modules) are limited to transition times of $\sim 8 \mathrm{~ns}$. Pre-chopping in chopper 1 ensures that no partially chopped bunches result from the slower field transition time of chopper 2 . Each switch module will dissipate $\sim 40 \mathrm{~W}$ mean, with chopper 1 electrodes, BD1, 2, and 3 dissipating beam powers of $\sim 35, \sim 100, \sim 35$, and $\sim 4530 \mathrm{~W}$, respectively.

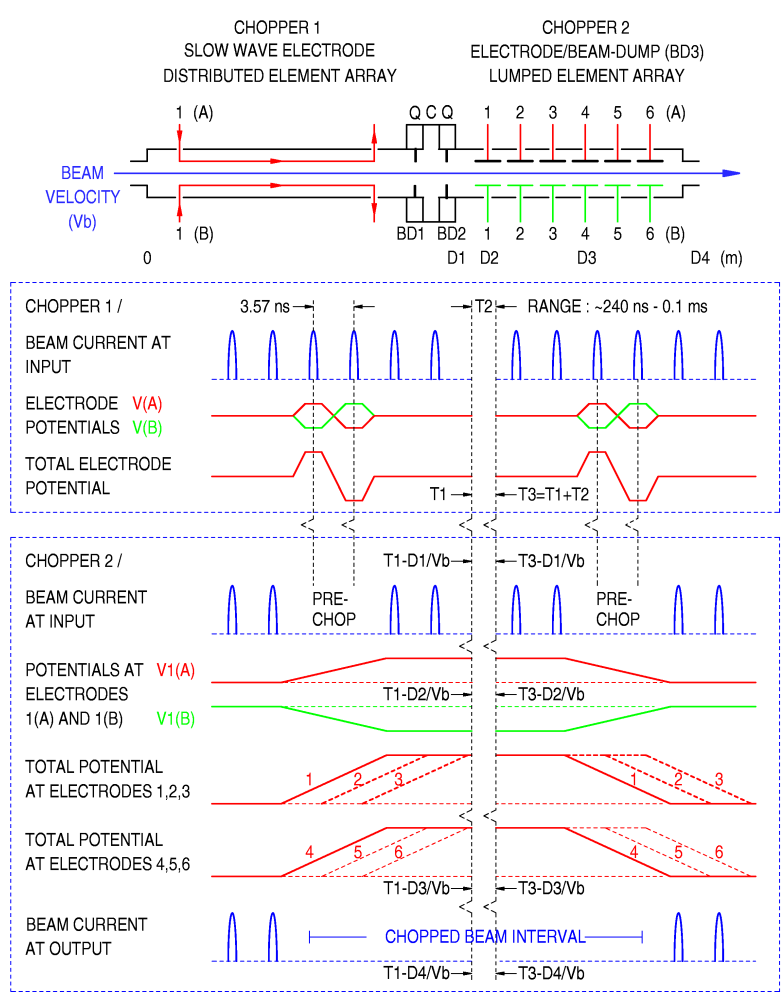

Figure 6: Scheme B / Timing schematic 


\section{MODULATOR DESIGN}

A block schematic of the proposed fast chopper modulator system, for modulation scheme $\mathrm{B}$, is shown in figure 7. Systems $1 \mathrm{~A}$ and $1 \mathrm{~B}$ drive chopper $1 \mathrm{~A}$ and $1 \mathrm{~B}$ distributed slow wave electrodes (see figure 4), and output fast transition ( $2 \mathrm{~ns})$, quasi-trapezoidal, bipolar high voltage pulses $( \pm 1.4 \mathrm{kV})$ into a $50 \mathrm{ohm}$ load. The modular configuration makes extensive use of high power transmission line transformers (TLT) [7] to impedance match, and combine the outputs from 32 solid state high voltage pulse generators.

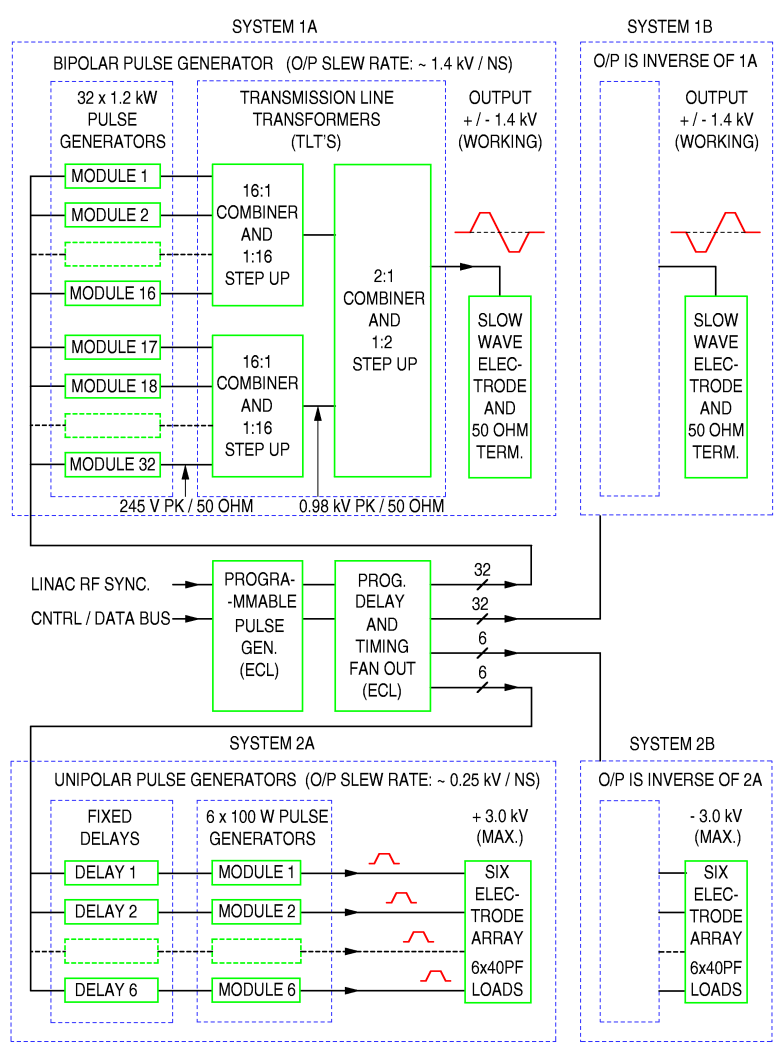

Figure 7: Scheme B / Modulator block schematic

A block schematic of the pulse generator module is shown in figure 8 . The class-D, push-pull, current switching design, outputs $\sim \pm 245 \mathrm{~V}$ (peak) from a $52 \mathrm{~V}$ supply using eight RF power mosfets $\left(150 \mathrm{~W}, 2 \mathrm{GHz}_{\mathrm{t}}\right)$,

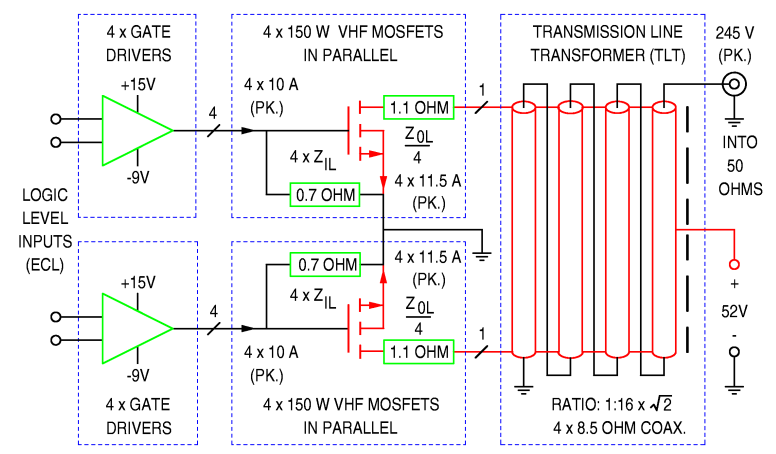

Figure 8: Scheme B / System 1 / Module

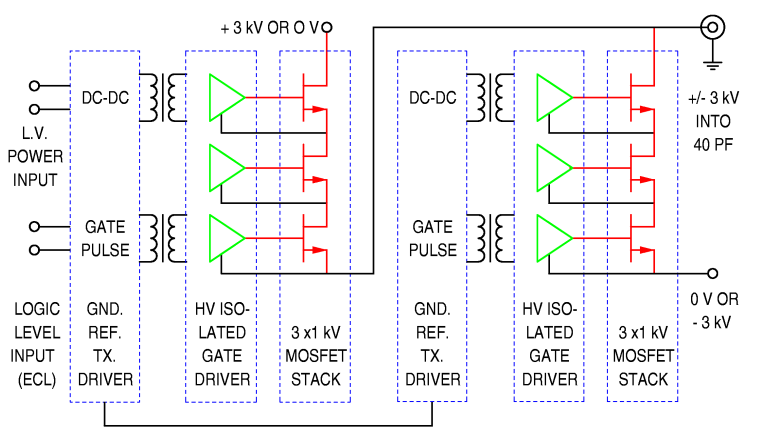

Figure 9: Scheme B / System 2 / Module

and a step up TLT. For scheme B, the module can be very compact, as the average duty cycle for system 1 is $\sim 0.12 \%$, and mean load power per module is only $\sim 1 \mathrm{~W}$.

Systems $2 \mathrm{~A}$ and $2 \mathrm{~B}$ drive chopper $2 \mathrm{~A}$ and $2 \mathrm{~B}$ lumped element slow wave electrodes, and output $\sim 8 \mathrm{~ns}$ transition, unipolar, trapezoidal pulses $(+3.0$ and $-3.0 \mathrm{kV})$ into a $40 \mathrm{pF}$ load. A block schematic of the module is shown in figure 9 . The high voltage mosfet switch has the standard dc-coupled, totem-pole configuration. The $40 \mathrm{~W}$ modules will be close-coupled $(<\lambda / 10=<50 \mathrm{cms})$ to individual electrodes to preserve signal integrity.

\section{SUMMARY}

A modulation scheme and a modulator design for the ESS $2.5 \mathrm{MeV}$ fast beam chopper have been identified. The scheme makes use of a new modulation technique that enables the implementation of an elegant, low average power, modulator.

\section{ACKNOWLEDGEMENT}

The author would like to thank Chris Prior for providing data for the beam envelope trajectories, presented in figures 3 and 5, and also Grahame Rees, and Ian Gardner, for their support, and encouragement.

\section{REFERENCES}

[1] ESS Volume III, 'The ESS Technical Study', Report No: ESS-96-53-M, 1996, ISBN 0902376 500/659.

[2] Spallation Neutron Source, (SNS), ORNL, USA; Japanese Hadron Facility, (JHF), KEK, Japan; Austron, Austria / CERN Switzerland.

[3] S.S. Kurennoy and J.F. Power, 'Development of Meander-Line current structure for SNS fast $2.5 \mathrm{MeV}$ Beam Chopper', Proc. of the 7th European Particle Accelerator Conference, Vienna, Austria, 2000, p.336-338.

[4] J.S. Lunsford and R.A. Hardekopf, 'Pulsed beam chopper for the PSR at LAMPF', IEEE Trans. Nucl. Sci., NS-30, 1983.

[6] C.R. Prior, 'Transverse image effects in the SNS and ESS beam choppers', RAL internal communication.

[7] E. Rotholtz, 'Transmission Line Transformers' IEEE Trans. Microwave Theory and Tech. MTT-29, No. 4 1981, p.327-331. 